\title{
Radiological evaluation in patients with clinical suspicion of cerebral venous sinus thrombosis presenting with nontraumatic headache - a retrospective observational study with a validation cohort
}

\author{
Håkan Almqvist ${ }^{1,2}$, Michael Mazya ${ }^{1,3}$, Alberto Falk Delgado ${ }^{4}$ and Anna Falk Delgado ${ }^{1,2^{*}}$ (i)
}

\begin{abstract}
Background: Clinical suspicion of cerebral venous sinus thrombosis (CVST) is imprecise due to non-specific symptoms such as headache. The aim was to retrospectively assess the diagnostic value of nonenhanced $C T$ (neCT) in patients with nontraumatic headache and clinically suspected CVST.

Methods: A retrospective consecutive series of patients referred 2013-2015 for radiology were evaluated. Eligible patients had nontraumatic headache and suspicion of CVST stated in the referral, investigated with CT venography (CTV) and nonenhanced CT (neCT). neCT scans were re-evaluated for the presence of CVST or other pathology. All CTVs were checked for the presence of CVST. The validation cohort consisted of 10 patients with nontraumatic CVT (2017-2019).

Results: Less than 1\% (1/104) had a suspected thrombus on neCT, confirmed by subsequent CTV. The remaining 99\% had a CTV excluding CVST. Eleven percent had other imaging findings explaining their symptoms. In the patient with CVST, the thrombosed dural sinus was high attenuating (maximum HU 89) leading to the suspicion of CVST confirmed by CTV. The validation cohort $(n=10)$ confirmed the presence of a high attenuating $(H U>65)$ venous structure in the presence of a confirmed thrombus in all patients presenting within 10 days (suspicion written in referral, 10\%).

Conclusions: Despite clinical suspicion, imaging findings of CVST in nontraumatic headache are uncommon. Evaluating neCT for high attenuation in dural sinuses, followed by CTV for confirmation in selected cases seems reasonable. CVST should be recognized by all radiologists and requires a high level of awareness when reading neCT for other indications.
\end{abstract}

Keywords: Cerebral venous sinus thrombosis (CVST), Diagnostic accuracy, Headache, High-attenuating, Nonenhanced computed tomography

\footnotetext{
* Correspondence: anna.falk-delgado@neuroradkarolinska.se

${ }^{1}$ Department of Clinical Neuroscience, Karolinska Institutet, 17176 Stockholm,

Solna, Sweden

${ }^{2}$ Department of Neuroradiology, Karolinska University Hospital, 17176

Stockholm, Solna, Sweden

Full list of author information is available at the end of the article
}

(c) The Author(s). 2020 Open Access This article is distributed under the terms of the Creative Commons Attribution 4.0 International License (http://creativecommons.org/licenses/by/4.0/), which permits unrestricted use, distribution, and reproduction in any medium, provided you give appropriate credit to the original author(s) and the source, provide a link to the Creative Commons license, and indicate if changes were made. The Creative Commons Public Domain Dedication waiver (http://creativecommons.org/publicdomain/zero/1.0/) applies to the data made available in this article, unless otherwise stated. 


\section{Background}

Cerebral venous sinus thrombosis (CVST) is an uncommon disease mainly occurring in patients with specific risk factors, such as inborn or acquired coagulopathies and trauma [1-3]. CVST affects fewer than two individuals per 100,000 persons/year [4, 5] with headache as the most common symptom, present in up to $89 \%$ of patients [2, 6-10]. The clinical diagnosis is difficult, with radiological investigation required to confirm the diagnosis [10-16]. Correct clinical suspicion of CVST is complicated by the fact that the condition frequently causes symptoms also seen in more common diagnoses, such as stroke or brain tumors. Further, more benign conditions such as idiopathic intracranial hypertension can mimic symptoms of CVST.

Isolated headache is a common but unspecific symptom for patients seen in emergency departments and only a fraction of these patients have CVST. Specifically, patients presenting with acute headache at the emergency department are often young women [17]. Patient age is one important consideration when deciding imaging strategy, since computed tomography $(\mathrm{CT})$ exposes the patient to ionizing radiation [18]. Previous studies have reported on the diagnostic utility of nonenhanced head computed tomography (neCT) to suspect CVST due to high density in the sinuses [11, 12, 19-22]. Despite previous reports suggesting the applicability of neCT to accurately identify acute CVST, other methods such as magnetic resonance imaging (MRI) or CT venography (CTV) are recommended to verify the diagnosis of CVST. Further, the use of a contrast-enhanced CTV only, would give incomplete evaluation of the cerebral parenchyma. MRI is a sensitive method for the evaluation of both brain parenchyma and cerebral vessels, but its limited availability for this indication restricts its potential to be a first order examination in many centers. Furthermore, MRI has a higher propensity for false positive diagnosis, with lower specificity [23]. Although considered the gold standard, digital subtraction angiography is not a common diagnostic tool for CVST.

At our department, patients with acute nontraumatic headache and a written referral to radiology asking to rule out or confirm CVST are primarily investigated with neCT with or without subsequent CTV, depending on the findings on neCT and the level of clinical suspicion for CVST. The level of clinical suspicion is based on clinical findings such as increased intracranial pressure, papilla oedema and known risk factors for CVST. The primary aim of this retrospective study was to assess imaging findings in patients with nontraumatic headache, a clinical suspicion of CVST primarily investigated with a neCT and subsequent CTV scans to establish the role for neCT as a screening tool for CVST. Study results were validated against imaging findings in a cohort of patients with confirmed CVT.

\section{Methods}

This study was approved by the regional ethical review board and informed consent by study participants was waived due to the retrospective nature of the study. Consecutive CT scans of patients referred to a high volume neuroradiological department were extracted from the Picture Archiving and Communication System (PACS), and Radiology Information System (RIS) from January 2013 to December 2015. Patients were presenting to the emergency department staffed by specialists and residents in neurology. The retrospective patient cohort was validated against a cohort of ten patients with confirmed cerebral venous thrombosis (CVT) investigated between 2017 and 2019.

\section{Main cohort \\ Eligibility criteria}

- Patient with a written imaging referral asking to rule out or confirm CVST in non-traumatic headache

- Radiological evaluation with neCT and CTV of the brain 2013-2015

- First time radiological examination for suspicion of CVST, onsite

\section{Exclusion criteria}

- Recent history of trauma or cervical, cranial or facial surgery (within 3 months)

- History of previous CVST

\section{Validation cohort \\ Eligibility criteria}

- CVT or CVST

- neCT and CTV of the brain 2017-2019 performed for any indication

- Radiological examination onsite or offsite (transferred images e $g$ due to patient transfer to our centre, or for radiological secondary review)

- Headache or other symptoms

\section{Exclusion criteria}

- Recent history of trauma or cervical, cranial or facial surgery (within 3 months)

\section{CT scan and blinded re-evaluation}

neCT examinations were performed with a General Electric CT 750 High definition, 64-slice scanner with 
$120 \mathrm{kV}$, CT dose index volume $(16 \mathrm{~cm})$, approximately 45 mGy-cm, soft algorithm and 30\% adaptive statistical iterative reconstruction (General Electric Healthcare, US, Chicago). Helical scanning mode, collimation $20 \mathrm{~mm}$, pitch 0 : 5 , rotation time $0.5 \mathrm{~s}, 0.625 \mathrm{~mm}$ slices without overlap, soft algorithm together with 3 plane reconstructions $5 \mathrm{~mm}$ without overlap saved routinely in the PACS. CTV was performed by injecting $100 \mathrm{cc}$ of iodine contrast (iodexanol $320 \mathrm{mg} \mathrm{I} / \mathrm{mL}$ ) at $4 \mathrm{cc} / \mathrm{sec}$ followed by $80 \mathrm{cc}$ of saline chaser. Craniocaudal scanning from vertex to the disk C2/ C3. Submillimetre slice with 50\% overlap $(0.625 / 0.315)$. Helical scan $0.4 \mathrm{~s}$ rotation, $80 \mathrm{kVp}$, table movement 0:984, automA function with $175-420 \mathrm{~mA}$ at noise index 22 . CTDIvol $_{16}$ between 8 and $20 \mathrm{mGy}$. Dose-length-product (DLP) between 270 and $500 \mathrm{mGycm}$ and DLP for the smartprep 10-35 mGycm. Manual start with minimum delay when the veins are clearly visible below the skull base with so called smart-prep technique.

Blinded for previously written report and patient symptoms, nonenhanced brain CT was re-evaluated in the local PACS by a specialist in Neuroradiology with 9 years of experience in brain CT evaluation. Scans were visually re-assessed for high density in cerebral dural sinuses on axial $0.63 \mathrm{~mm}$ thin slices and on reformatted 5 $\mathrm{mm}$ thick sagittal, axial, and coronal slices. Scans were evaluated for the presence of a suspected CVST or other imaging findings, including the presence of thrombus in the deep venous system. Visual inspection assessed heterogeneity in grey-scale within the intracranial venous structures and compared the grey-scale as appreciated in the dural sinuses compared to arterial structures and normal brain parenchyma. If a scan was reported positive for suspected CVST, a measurement of maximum Hounsfield units (HUs) in the suspected clot was also performed. Indeterminate test results were discussed with a second investigator with 16 years of experience in neuroradiology.

\section{Region of interest delineation}

Standardized measurements of $\mathrm{HU}$ in cerebral dural sinuses were performed in the torcular herophili, superior sagittal sinus, transversal sinus, and sigmoid sinus bilaterally as shown in Fig. 1a-d in all included patients. In region of interest delineation, care was taken to avoid intravenous Paccioni granulations, dural structures, and beam hardening artifacts from nearby skull bone. Exact ROI position is detailed in the figure legends.

\section{Patient data extraction}

After visual and quantitative evaluation of scans, the original radiology report was controlled for congruency with the blinded re-evaluation. In clinical routine, each original report had been double-signed by at least one specialist in general radiology and one experienced neuroradiologist. CTV findings and final diagnosis from patient charts were tabulated. Data on patient sex, age, and clinical information on risk factors for CVST were extracted from patient records. Laboratory reports were checked for hemoglobin and d-dimer values at the time of the radiological examination.

\section{Statistical analysis}

Descriptive statistical methods were applied to summarize the extracted data. Data points were visually presented as scatter and box plots. Correlation analysis was performed with the Pearson's correlation test. Statistical differences between groups were evaluated with Pearson's Chi-2, Fisher's exact test and Student T-test as appropriate. Receiver operating analysis was performed to assess diagnostic performance, optimal cut-off and sensitivity and specificity. Statistical analyses were performed in Statistica 12 (Dell Inc., Tulsa, OK, USA).

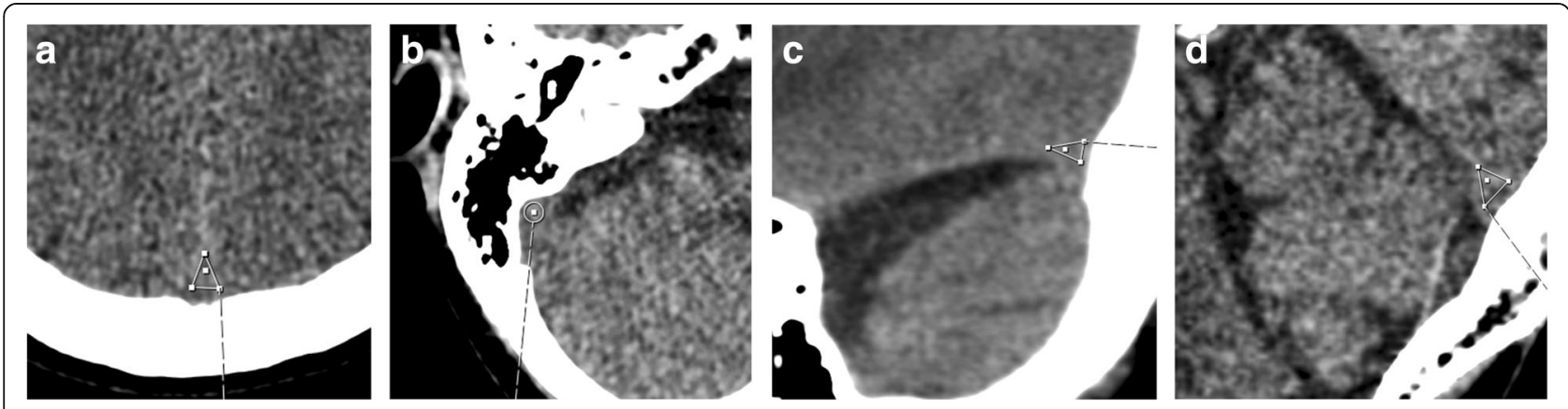

Fig. 1 a Region of interest delineation in the superior sagittal sinus was performed on $0.63 \mathrm{~mm}$ thin axial slices at the level above the lateral ventricles as a triangular shape in the sinus. $\mathbf{b}$ Region of interest delineation in the left and right sigmoid sinus was performed on axial 0.63 mm thick slices at the level of the internal acoustic meatus in the sinus. Red arrow indicates region of measurement. c $A$ region of interest (ROI) in the left and right transverse sinus was performed on reformatted $5 \mathrm{~mm}$ sagittal slices at the level just lateral to the lateral ventricles as a triangular shaped ROI. Red arrow indicates region of measurement. $\mathbf{d}$ A region of interest (ROI) in the torcular herophili was delineated on sagittal reformatted $0.63 \mathrm{~mm}$ thick slices 


\section{Results}

\section{Patient characteristics}

In total, 104 patients (84 females, $81 \%$ ) were included in the main study cohort. Mean (SD) patient age was 37 (12) years. Median (IQR) duration of headache was 7 (2-21) days. All patients in the main study cohort had been referred for a neuroradiological examination, been examined with both a neCT and CTV, had a medical history of nontraumatic headache, and a clinical suspicion from the referring physician of CVST written in the radiological referral. Referrals for head CT examination were from the emergency department in 81 cases (78\%), inpatient wards in 18 cases (17\%) and the outpatient neurology clinic in 5 cases $(5 \%)$.

Ten patients were included in the validation cohort. Sixty percent $(6 / 10)$ were evaluated at the same hospital as the main cohort while $40 \%(4 / 10)$ had their primary investigation in an outside hospital, with images digitally transferred for a second radiological review. Mean (SD) age was 47 (28) years ( $p \geq 0.05$ versus the main cohort). Six patients $(60 \%)$ were female.

The proportion of females did not differ significantly between the main and validation cohort, $p=0.13$.

\section{Symptom duration and risk factors main cohort}

CVST $(n=1)$ was related to a symptom duration of headache for 3 days compared to unaffected individuals $(n=103)$, who had a median (IQR) symptom duration of headache of 7 (2-21) days. Out of 104 patients, 47\% (49/ 104) had no known risk factor for venous thromboembolism (VTE) in the medical records or radiological referral. Fifty-three percent $(55 / 104)$ had one or more reported risk factor of: current pregnancy $(n=2)$, recent pregnancy $(n=15)$, treatment with oral contraceptives $(n=3)$, ongoing in-vitro fertilization treatment $(n=1)$, history of VTE $(n=12)$, recent or current facial/ear/ meningeal infection $(n=10)$, current oncological disease $(n=2)$, high hematocrit or polycythemia vera $(n=2)$, antiphospholipid syndrome $(n=4)$, Von Willebrand's disease $(n=1)$, thalassemia minor $(n=1)$, heredity for pulmonary embolism $(n=1)$, and systemic inflammatory disease $(n=3)$. All included patients had imaging performed to rule out or confirm clinically suspected CVST.

\section{Symptom duration and risk factors, validation cohort}

Median (IQR) symptom duration was 3 (1-7) days. Sixty percent $(6 / 10)$ presented with headache. Other symptoms were loss of consciousness $(n=2)$, seizures $(n=1)$ and aphasia $(n=1)$. Only one patient $(1 / 10(10 \%))$ had a clinical suspicion of CVST written in the referral (Table 2). The queries on the referrals included: hemorrhage? $(n=6)$, infarct? $(n=4)$, tumor? $(n=3)$, CVST? $(\mathrm{n}=1)$, and dissection? $(n=1)$. Seventy percent had a known risk factor for thromboembolic events, compared to $58 \%$ in the main cohort $(p=0.34)$.

\section{$\mathrm{NeCT}$ and $\mathrm{HU}$ in unaffected and thrombosed sinuses, main cohort}

In blinded reevaluation, one patient $(1 / 104,1.0 \%)$ had suspicion of CVST presenting as an intradural high density (Fig. 2) with the highest HU in the left sigmoid sinus $(\mathrm{HU}=89)$, while 103 patients $(99.0 \%)$ had no suspicion of CVST on neCT.

Data on HU in unaffected sinuses in the 103 CVSTnegative patients is presented in Table 1 . Median HU in the torcular herophili was 52.0 (IQR 48.0-55.0), superior sagittal sinus 57 (IQR 54-60), 59 in left (IQR 55-64) and right (IQR 54-63) sigmoid sinuses and 57 (IQR 5460) and 56 (IQR 54-59) in left and right transverse sinus. The maximum $\mathrm{HU}$ in the patient with suspected thrombus on neCT was 89 in the thrombus measured in the left sigmoid sinus (CVST confirmed by CTV as depicted in Fig. 3). An inverse correlation between $\mathrm{HU}$ and patient age was found, with HU decreasing with increasing age $(r=-0.20, p=0.04)$. The variance of $\mathrm{HU}$ was highest in the sigmoid sinus (6.1-6.6 HU) and lowest in transverse sinuses and torcular herophili (4.1-4.5 $\mathrm{HU})$.

\section{$\mathrm{NeCT}$ and $\mathrm{HU}$ in unaffected and thrombosed sinuses, validation cohort}

In the validation cohort, unblinded reevaluation confirmed the presence of a hyperdense sinus in all patients but one. Including the validation cohort, 11 patients had a venous thrombosis with a mean HU of 75.0 (SD 4.9) measured in the thrombus, compared to 51.7 (4.4) HU measured in the torcular herophili of unaffected individuals $(p<0.01)$ (Fig. 4). Figure 5 describes the non-linear

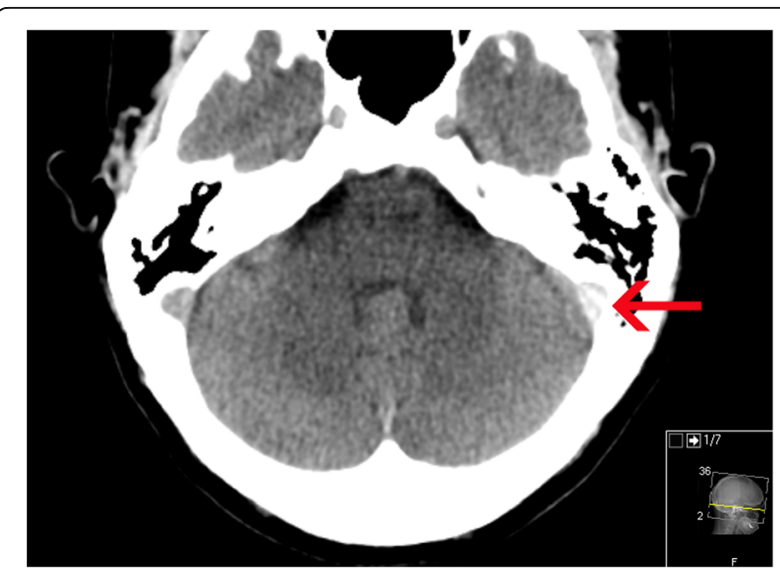

Fig. 2 Axial reformatted $5 \mathrm{~mm}$ nonenhanced $C T$ of suspected thrombus in the left sigmoid sinus marked by a red arrow (window width 90 window level 40). Note the normal $\mathrm{HU}$ value in the corresponding right sigmoid sinus 
Table 1 Hounsfield unit values from nonenhanced CT in dural sinuses in patients $(n=103)$ not diagnosed with cerebral sinus venous thrombosis

\begin{tabular}{lllll}
\hline Descriptive Statistics & Mean (SD) & Median (IQR) & Minimum & Maximum \\
\hline Hounsfield units in torcular herophili & $51.7(4.4)$ & $52.0(48.0-55.0)$ & 40.0 & 39.0 \\
Hounsfield units in superior sagittal sinus & $56.6(4.7)$ & $57.0(54.0-60.0)$ & 59.0 & 68.0 \\
Hounsfield units in left sigmoid sinus & $58.5(6.6)$ & $59.0(54.0-64.0)$ & 40.0 & 69.0 \\
Hounsfield units in right sigmoid sinus & $58.3(6.1)$ & $57.0(54.0-60.0)$ & 46.0 & 40.0 \\
Hounsfield units in left transverse sinus & $56.9(4.1)$ & $56.0(54.0-59.0)$ & 40.0 & 66.0 \\
Hounsfield units in right transverse sinus & $56.0(4.5)$ & & & 64.0 \\
\hline
\end{tabular}

relationship between $\mathrm{HU}$ and symptom onset. Receiver operating characteristic analysis using the highest measured HU in the thrombosed region $(n=11)$ against torcular herophili $\mathrm{HU}$ in unaffected patients $(n=103)$ revealed maximum accuracy at cut-off HU 65 (AUC = $1)$. This discriminated between thrombus and normal sinus with $100 \%$ sensitivity and specificity.

\section{CTV, main cohort}

All included patients underwent CTV in addition to neCT of the brain. CTV was performed on the same day in 97 patients (93\%), in three patients within 1 week, two patients within 2 months and one patient after 8 months and one patient after 22 months. CTV was only positive for CVST in the one patient with suspected CVST on initial neCT (Fig. 2) with thrombus in the left transverse and sigmoid sinus. At a retrospective followup of medical charts (median duration of follow-up 29 months, IQR 21-32), three patients were deceased, two from cancer and one due to drug abuse. There were no additional patients diagnosed with CVST or readmitted due to CVST during this follow-up period. The single patient with CVST on CTV in the main study cohort, made a rapid recovery following start of medical

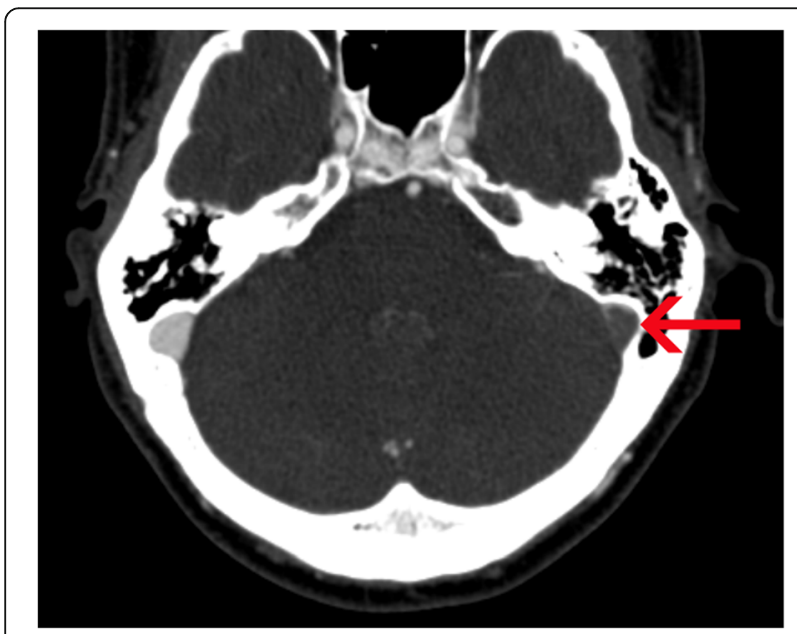

Fig. 3 Axial multiplanar reconstruction ( $2 \mathrm{~mm}$ ) of CTV verifying the thrombosed left sigmoid sinus indicated by a red arrow treatment, with a complete remission of headache within 2 days. Follow-up CTV after 7 months showed complete restoration of venous flow in the affected sinuses.

\section{CTV, validation cohort}

CTV images were retrospectively assessed for the presence of CVT and confirmed in all cases. One patient was confirmed to have CVST by MRV. All patients in the validation cohort had non-traumatic CVT confirmed by $\operatorname{CTV}(n=9)$ or MRV $(n=1)$ (Table 2$)$.

\section{Other imaging findings and final diagnosis, main cohort} In addition to the single patient with CVST, there were eleven additional cases with pathological findings on CT imaging: subarachnoid and subdural hemorrhages were seen in two patients each, meningioma, cerebral metastases, cerebral infarct, arterial aneurysm, and suspected Chiari malformation in one patient each, as well as two cases with fluid levels in the sphenoid sinus indicating sinusitis. Ninety-two (88\%) patients had no pathological imaging findings.

\section{Other imaging findings and final diagnosis, validation cohort}

Besides the presence of CVT, two patients had focal parenchymal edema and one patient had generalized parenchymal edema. Three patients had intracerebral hemorrhage and one had cerebral metastasis. The remaining three patients had no additional imaging findings (Table 2).

\section{Laboratory findings and lumbar pressure, main cohort} Hemoglobin $(\mathrm{Hb})$ ranged from 89 to $176 \mathrm{~g} / \mathrm{L}$, mean 135 g/L (SD 16). Hb did not significantly correlate with HU in the superior sagittal sinus $(r=0.17, P=0.09)$ in patients without CVST $(n=103)$. Thirteen patients had been investigated with a D-dimer test, of which 8 had elevated levels $(\geq 0.30 \mathrm{mg} / \mathrm{L})$. One of the patients with elevated D-dimer $(0.30 \mathrm{mg} / \mathrm{L})$ had confirmed CVST on CTV. The mean levels in patients with elevated D-dimer were $1.8(3.5) \mathrm{mg} / \mathrm{L}$, range $0.3-10.5$. Thirty-one patients had been subject to a lumbar puncture including lumbar cerebrospinal fluid (CSF) pressure measurement. The 


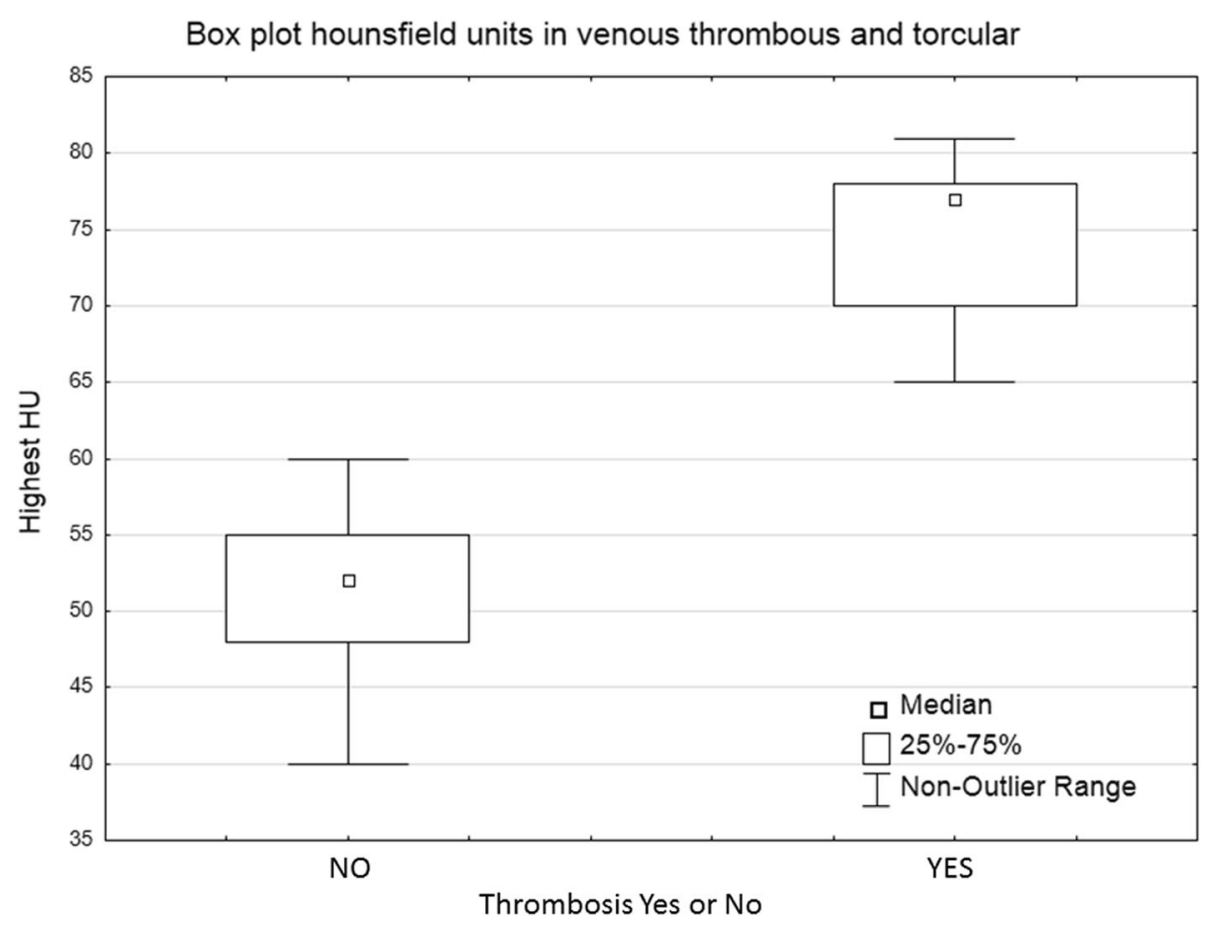

Fig. 4 Box plot of hounsfield units measured in the thrombus (maximum HU) and in unaffected torcular herophili

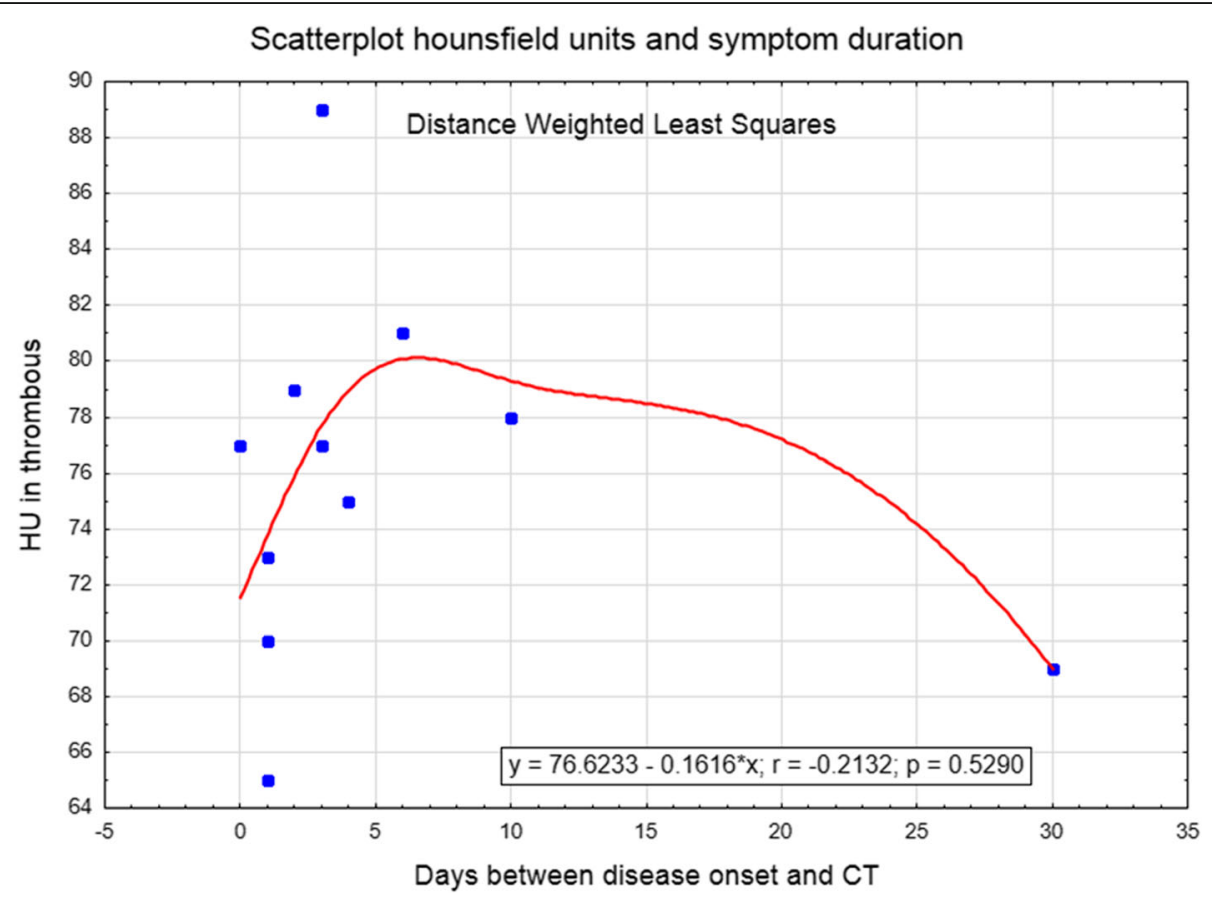

Fig. 5 Scatterplot with $\mathrm{HU}$ measured in the thrombus ( $y$-axis) and days between symptom onset and neCT ( $x$-axis). A non-linear relationship between symptom duration and thrombus density is noted. HU increases over the first days 


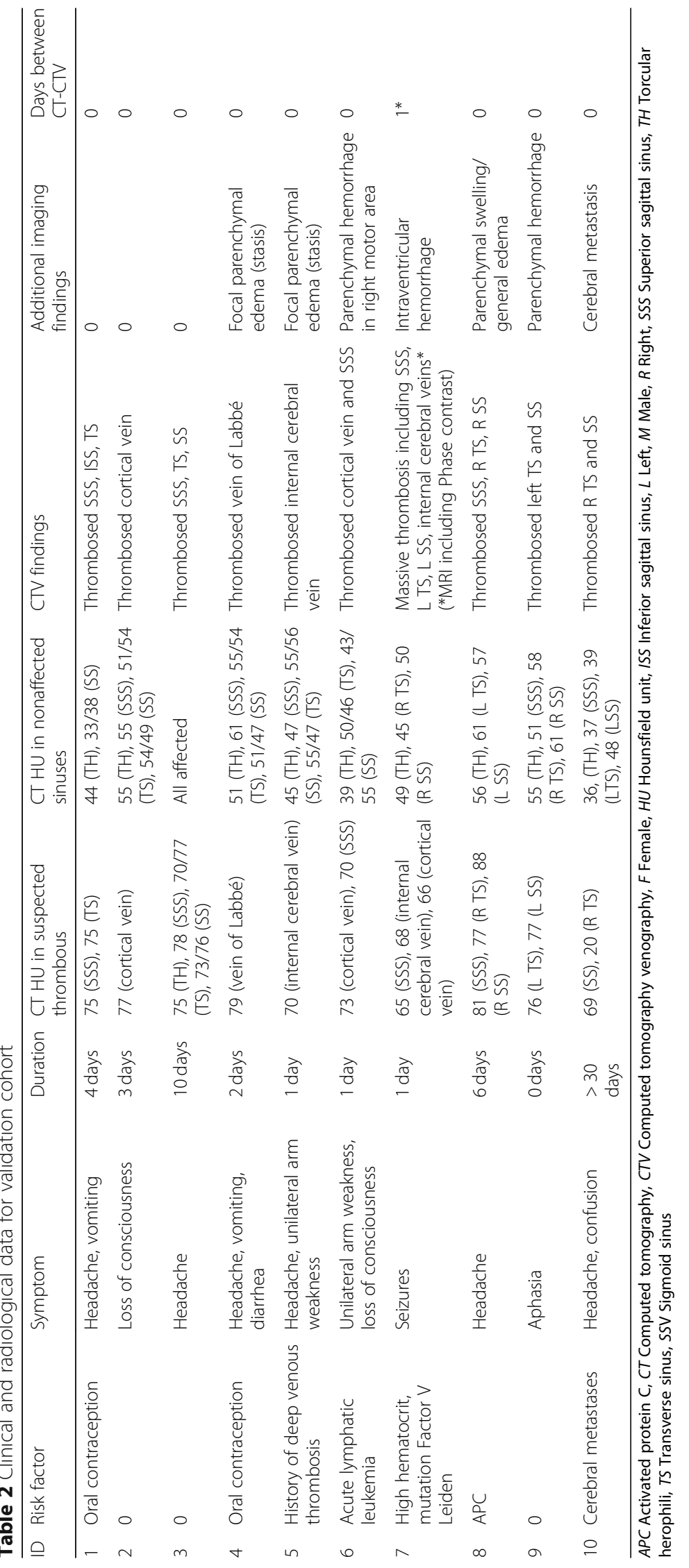


lumbar pressure ranged from 7 to $50 \mathrm{~cm} \mathrm{H}_{2} \mathrm{O}$. Twentyone patients had a lumbar pressure above $20 \mathrm{~cm} \mathrm{H}_{2} \mathrm{O}$. In this material, the patient with confirmed CVST did not undergo a lumbar puncture.

\section{Clinical diagnosis, main cohort}

Diagnosis at discharge was unspecified headache in $44 \%$ of patients (46/104), tension headache in 19\% (20/104), migraine in $8 \%(8 / 104)$, idiopathic intracranial hypertension in $8 \%(8 / 104)$ and one patient each in the following categories: ischemic stroke, depression, post lumbarpuncture headache, reversible cerebral vasoconstriction syndrome, sepsis, sinusitis, dacrocystitis, upper respiratory tract infection, viral meningitis, unspecified virosis, Chiari type 1, intracranial hypotension, cerebral metastasis, jugular vein thrombosis below the level of $\mathrm{C} 2$, and two patients with undefined diagnosis.

\section{Discussion}

This retrospective study of imaging findings in patients with nontraumatic headache and a clinical suspicion of CVST, justifies neCT as a primary screening tool for intracranial pathology. In our study, we found no cases of CT venography-verified CVST among patients with normal attenuation of venous sinuses on neCT and symptom duration less than 7 days. In the validation group, one patient with symptoms longer than 30 days had low attenuation $(20 \mathrm{HU})$ in parts of the thrombosed dural sinuses. Our results also advise against using primary $\mathrm{CT}$ venography without neCT, since neCT showed other pathological findings in $11 \%$ of cases.

The main strength of this study is that it reflects a true clinical situation, including consecutive patients with nontraumatic headache and a clinical suspicion of CVST at one center over 3 years, investigated under similar conditions. The study shows a high incidence of negative radiological investigations. The use of $\mathrm{D}$-dimer as a negative predictor and an adjunct to radiology in patients with suspected CVST has been recommended in the recently published European Stroke Organisation guidelines for the management of CVST [24]. Meanwhile, this recommendation was given with an important caveat regarding patients with isolated headache, or symptoms persisting for more than 1 week before the workup, as both factors have been associated with falsenegative D-dimer results [24]. Furthermore, data on HU values from standardized measurements in patients without CVST are presented. Our study gives a reference HU range based on 103 patients with similar symptoms and clinical suspicion of CVST with confirmed negative CTV.

The finding of hyperattenuation in a thrombosed sinus is in accordance with previously reported findings [11, 12, 19-21]. In previously published material different cutoffs for $\mathrm{HU}$ have been proposed ranging from 62 to $70[12,19-21]$. Data of non affected sinuses in this study can aid radiological decision-making when trying to rule-out or lower the suspicion of CVST. The mean HU value in non affected sinuses was 56.9 (SD 5.1) and the optimal cut-off was $65 \mathrm{HU}$.

Differences in measured mean HU between studies can be explained by differences in population selection, hematocrit level, type and calibration of CT-scanner, and region of interest delineation method. Our results do not indicate a strong correlation between hemoglobin counts and $\mathrm{HU}$ in the superior sagittal sinus. However, we found a negative correlation between $\mathrm{HU}$ and patient age. This study suggests that primary use of CTV without prior neCT might lead to missed other diagnoses.

Our study is limited by its single-center, retrospective design and the findings would benefit from confirmation in prospective multi-center studies. Our results cannot be generalized to patients with headache after head trauma, nor to patients with recurrent headache after previous CVST, as such cases were excluded. Due to the retrospective nature of the data, we could not reassess the criteria for when the referring physician should ask to rule out or confirm CVST. Only one radiologist measured the $\mathrm{HU}$, limiting the possibility for interobserver variance to be assessed. However, the intraobserver variance of measured $\mathrm{HU}$ within normal regions was lower than the difference in HU between CVST and not CVST regions, likely restricting the impact of this limitation. Inclusion criteria differed between the main cohort and the validation cohort allowing to include more patients with CVT in the validation group. The main reasons for a higher proportion of patients with thrombosis in the validation group were: no requirement for the referring physician to suspect CVST in the written referral, no requirement for the patient to present with headache, and the inclusion of deep and cortical CVT, as well as transferred images from other centra.

\section{Conclusions}

Despite clinical suspicion, imaging findings of CVST in nontraumatic headache are uncommon. Evaluating neCT for high attenuation in dural sinuses, followed by CTV for confirmation in selected cases seems reasonable. CVST should be recognized by all radiologists and requires a high level of awareness when reading neCT for other indications.

\section{Abbreviations}

CT: Computed tomography; CTV: Computed tomography venography; CVST: Cerebral venous sinus thrombosis; CVT: Cerebral venous thrombosis; HU: Hounsfield unit; MRI: Magnetic resonance imaging; neCT: nonenhanced CT; PACS: Picture archiving and communication system; RIS: Radiology Information System; VTE: Venous thromboembolism 


\section{Acknowledgements}

Jenny Rydén for proofreading.

\section{Authors contribution}

$\mathrm{HA}, \mathrm{AFD}, \mathrm{AFD}$ and MM contributed to the design and interpretation of the study findings. HA retrieved the data. AFD analyzed the data and drafted the manuscript. HA, AFD, and MM critically revised the manuscript. All authors read and approved the final manuscript.

\section{Funding}

An.FD. received financial support from the Neuroradiological Department, Karolinska University Hospital. Michael Mazya was supported by the Stockholm County Council (clinical postdoctoral appointment). The funding sources had no role in the study: Unrelated: Karolinska University Hospital has research collaboration with Philips Healthcare and GE Healthcare.

\section{Availability of data and materials}

Data presented in this article can be made available upon reasonable request to: anna.falk-delgado@neuroradkarolinska.se

\section{Ethics approval and consent to participate}

This work has been carried out with ethics approval from the ethical review board in Stockholm (Etikprövningsmyndigheten Stockholm) DNR 2017/198331/2. The need for patient consent was waived.

\section{Consent for publication}

Not applicable.

\section{Competing interests}

The authors declare that they have no competing interests.

\section{Author details}

'Department of Clinical Neuroscience, Karolinska Institutet, 17176 Stockholm, Solna, Sweden. ${ }^{2}$ Department of Neuroradiology, Karolinska University Hospital, 17176 Stockholm, Solna, Sweden. ${ }^{3}$ Department of Neurology, Karolinska University Hospital, Stockholm, Sweden. ${ }^{4}$ Department of Surgical Sciences, Uppsala University, Uppsala, Sweden.

Received: 11 September 2019 Accepted: 19 February 2020 Published online: 27 February 2020

\section{References}

1. Narayan D, Kaul S, Ravishankar K, et al. Risk factors, clinical profile, and longterm outcome of 428 patients of cerebral sinus venous thrombosis: insights from Nizam's institute venous stroke registry, Hyderabad (India). Neurol India. 2012;60:154-9

2. Terni E, Giannini N, Chiti A, et al. Cerebral sinus venous thrombosis: clinical and pathogenetic perspectives from Tuscany. Blood Coagul Fibrinolysis. 2015;26:505-8

3. Tatlisumak T, Jood K, Putaala J. Cerebral venous thrombosis: epidemiology in change. Stroke. 2016;47:2169-70.

4. Devasagayam S, Wyatt B, Leyden J, Kleinig T. Cerebral venous sinus thrombosis incidence is higher than previously thought: a retrospective population-based study. Stroke. 2016:47:2180-2.

5. Coutinho Jm, Zuurbier Sm, Aramideh M, Stam J. The incidence of cerebral venous thrombosis: a cross-sectional study. Stroke. 2012;43:3375-7.

6. Mortimer Am, Bradley Md, Stoodley Ng, Renowden Sa. Thunderclap headache: diagnostic considerations and neuroimaging features. Clin Radiol. 2013;68:e101-13

7. Sparaco M, Feleppa M, Me B. Cerebral venous thrombosis and headache--a case-series. Headache. 2015:55:806-14.

8. Wittmann $M$, Dewald D, Urbach $H$, et al. Sinus venous thrombosis: a differential diagnosis of postpartum headache. Arch Gynecol Obstet. 2012; 285:93-7.

9. Gameiro J, Ferro J, Canhao P, et al. Prognosis of cerebral vein thrombosis presenting as isolated headache: early vs. late diagnosis. Cephalalgia. 2012; 32:407-12.

10. Thammishetti $V$, Dharanipragada S, Basu D, Ananthakrishnan R, Surendiran D. A prospective study of the clinical profile, outcome and evaluation of Ddimer in cerebral venous thrombosis. J Clin Diagn Res. 2016;10:OC07-10.
11. Zaheer S, lancu D, Seppala N, et al. Quantitative nondiagnosticcontrast measurements improve diagnosing dural venous sinus thrombosis. Neuroradiology. 2016;58:657-63.

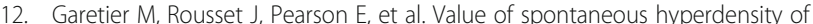
cerebral venous thrombosis on helical CT. Acta Radiol. 2014;55:1245-52.

13. Niu Pp, Yu Y, Guo ZN, et al. Diagnosis of nondiagnosticacute cerebral venous thrombosis with 3D T1-weighted black blood sequence at 3T. J Neurol Sci. 2016;367:46-50.

14. Patel $D$, Machnowska $M$, Symons $S$, et al. Diagnostic performance of routine brain MRI sequences for Dural venous sinus thrombosis. AJNR Am J Neuroradiol. 2016:37(11):2026.

15. Jalli $R$, Zarei F, Farahangiz $S$, et al. The sensitivity, specificity, and accuracy of contrast-enhanced $\mathrm{T} 1$-weighted image, $\mathrm{T}^{*}$-weighted image, and magnetic resonance venography in diagnosis of cerebral venous sinus thrombosis. J Stroke Cerebrovasc Dis. 2016;25:2083-6.

16. Gratma van Andel Ha, van Boven Lj, van Walderveen Ma, et al. Interobserver variability in the detection of cerebral venous thrombosis using CT venography with matched mask bone elimination. Clin Neurol Neurosurg. 2009;111:717-23.

17. Chu K, Te H, Keijzers $G$, et al. Acute headache presentations to the emergency department: a statewide cross-sectional study. Acad Emerg Med. 2017:24:53-62.

18. Bernier Mo, Rehel Jl, Brisse Hj, et al. Radiation exposure from CT in early childhood: a French large-scale multicentre study. Br J Radiol. 2012:85:53-60

19. Alsafi A, Lakhani A, Carlton Jones L, Lobotesis K. Cerebral venous sinus thrombosis, a nonenhanced CT diagnosis? Radiol Res Pract. 2015;2015:581437.

20. Black Df, Rad Ae, Gray La, Campeau Ng, Kallmes Df. Cerebral venous sinus density on noncontrast CT correlates with hematocrit. AJNR Am J Neuroradiol. 2011:32:1354-7.

21. Buyck Pj, De Keyzer F, Vanneste D, Wilms G, Thijs V, Demaerel P. CT density measurement and $\mathrm{H}: \mathrm{H}$ ratio are useful in diagnosing acute cerebral venous sinus thrombosis. AJNR Am J Neuroradiol. 2013;34:1568-72.

22. Roland T, Jacobs J, Rappaport A, Vanheste R, Wilms G, Demaerel P. Unenhanced brain $C T$ is useful to decide on further imaging in suspected venous sinus thrombosis. Clin Radiol. 2010;65:34-9.

23. Sadigh G, Mullins M, Saindane A. Diagnostic performance of MRI sequences for evaluation of Dural venous sinus thrombosis. AJR Am J Roentgenol. 2016;206:1298-306.

24. Ferro Jm, Bousser Mg, Canhao $P$, et al. European stroke organization guideline for the diagnosis and treatment of cerebral venous thrombosis - endorsed by the European academy of neurology. Eur J Neurol. 2017;24:1203-13.

\section{Publisher's Note}

Springer Nature remains neutral with regard to jurisdictional claims in published maps and institutional affiliations.

Ready to submit your research? Choose BMC and benefit from:

- fast, convenient online submission

- thorough peer review by experienced researchers in your field

- rapid publication on acceptance

- support for research data, including large and complex data types

- gold Open Access which fosters wider collaboration and increased citations

- maximum visibility for your research: over $100 \mathrm{M}$ website views per year

At $\mathrm{BMC}$, research is always in progress.

Learn more biomedcentral.com/submissions 